\title{
Gelehrtes Scheitern: Der parodistische Umgang mit Euripides in Lukians Werken
}

\begin{abstract}
This paper explores the usage of quotations from Euripides in Lucian's works with regard to the discussion of learnedness (paideia) in the Second Sophistic. As a means of both, displaying paideia and exposing the lack of it, quotations from the literary tradition not only help shape the characters, but often bear poetological meaning and show traces of generic enrichment leading to literary hybridity. Furthermore, by way of parody the learnedness of the recipients as well as traditional ways of reading are put to the test. Focussing on the Piscator, Juppiter Tragoedus, Necyomantia and Podagra, the role of quotations in reflecting upon literary tradition and creating literary innovation (comedian dialogue) will be analyzed.
\end{abstract}

In Lukians Schrift Adversus Indoctum führt ein anonymer Sprecher einem Büchernarren, der glaubt, dass allein schon der physische Besitz von Büchern ein Bildungsausweis sei, polemisch dessen Unbildung vor Augen; dabei erzählt er folgende Anekdote:

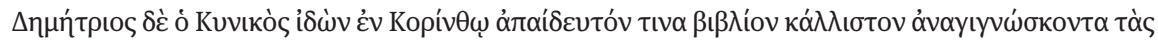

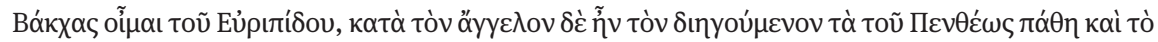

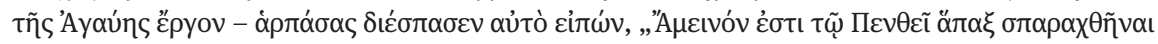

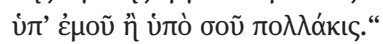

Der Kyniker Demetrios sah in Korinth einmal einen Ungebildeten ein großartiges Buch lesen - die Bakchen des Euripides, glaube ich, und er war gerade bei der Stelle, wo der Bote vom schrecklichen Tod des Pentheus und von der Tat der Agave berichtet - : Da schnappte er es sich, riss es in kleine Fetzen und sagte: „Besser für Pentheus, er wird einmal von mir zerrissen als viele Male von dir.“ (Ind. 19, Übs. v. Möllendorff 2006) ${ }^{1}$

Die Begebenheit, die im 1. Jh. n.Chr. zu Lebzeiten des Kynikers Demetrios, eines Zeitgenossen und Freundes von Seneca, spielt, ,bezeugt' die große Verbreitung von Euripides' Werken auf dem kaiserzeitlichen Buchmarkt ebenso wie sie ein Beispiel für die starke Bezugnahme auf seine Tragödien innerhalb des lukianischen Euvres ist. Beides erscheint zunächst wenig überraschend angesichts der Tatsache, dass Euripides in der Kaiserzeit als der Hauptvertreter der attischen Tragödie galt, ${ }^{2}$ vielfach im Schulunterricht gelesen wurde und für einen rhetorisch gebildeten Autor wie Lukian, bei dem man unabhängig von der Glaubwürdigkeit der hierzu gemachten ,autobio-

1 Soweit nicht anders angegeben, stammen die Übersetzungen in diesem Beitrag vom Verfasser.

2 Vgl. die Bezeichnung als ò траүıкó (TrGF 5,1, T 155-157a) und die Einleitung von Michael Schramm in diesem Band, S. 2. 
graphischen' Aussagen im Somnium oder im Bis Accusatus davon ausgehen darf, dass er eine ausgezeichnete Rhetorikausbildung gehabt haben muss, ${ }^{3}$ zum Rüstzeug gehörte. ${ }^{4}$

Entsprechend ist Euripides nach Homer $^{5}$ der mit Abstand am meisten zitierte Dichter in Lukians Werken, und er wird im Unterschied zu den insgesamt sieben Erwähnungen der beiden anderen kanonischen Tragiker, Aischylos (zweimal) und Sophokles (fünfmal), allein 20-mal namentlich genannt und über 50-mal zitiert bzw. parodiert. ${ }^{6}$ Dabei fällt auf, dass viele Euripides-Zitate nicht nur als bloße Bildungsausweise - sei es auf der Ebene der Figurenhandlung, sei es auf Seiten der (auktorial konnotierten) Erzähler - fungieren, sondern bestimmte Positionierungen im Bildungsdiskurs der Zeit erkennen lassen und von Lukian deutlich stärker als Bezüge zu anderen Autoren bzw. Werken zur Gestaltung und Reflexion von eigenen literarischen Innovationen genutzt werden.

\section{1 ,Euripides“ in aller Munde: Das Scheitern gelehrter Zitate auf der Figurenebene}

Im Bildungsdiskurs der so genannten Zweiten Sophistik, der wesentlich zwischen Philosophen, Rednern und Schriftstellern als den intellektuellen Eliten in der Zeit vom 1.-3. Jh. n.Chr. ${ }^{7}$ geführt wurde, standen Frage nach dem richtigen Umgang mit den kulturellen Errungenschaften und den philosophischen, rhetorischen und literarischen Traditionen im Mittelpunkt. Da Bildung ( $\left.\pi \alpha \_\delta \varepsilon^{\prime} \alpha\right)$ in der Antike fest mit der Vermittlung von Werten verbunden war, richtete sich der Blick immer auch auf ihren ethischen Nutzen für ein Individuum bzw. die Gesellschaft. ${ }^{8}$ Vor diesem Hintergrund

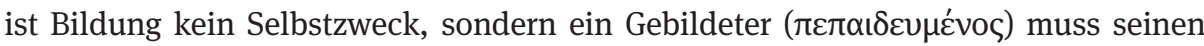
Bildungsanspruch immer wieder neu - besonders im Umgang mit anderen Gebildeten - unter Beweis stellen. Und dafür reicht es nicht, ,seinen` Euripides zu kennen oder

3 Hierfür legen allein mit Blick auf die Verwendung rhetorischer Gattungen die beiden Deklamationen Abdicatus (Der enterbte Sohn) und Tyrannicida (Der Tyrannenmörder) sowie die neun prolaliaí (vgl. Nesselrath 1990) und das paradoxe Lob der Fliege (Muscae Encomium) Zeugnis ab.

4 Zur Bedeutung der Tragödie bzw. von Euripides für die Rhetorikausbildung der Kaiserzeit vgl. Philostr. Vit. Soph. 620; Quint. 10,1,67f.

5 Vgl. hierzu die Arbeit von Bouquiaux-Simon 1968.

6 Dagegen finden sich nur sieben Zitate aus Sophokles und zwei aus Aischylos, vgl. zum statistischen Befund Karavas 2005, 137-170, der 58 von insgesamt 75 zitierten tragischen Versen im lukianischen Euvre Euripides zurechnet (ebd. 169), und Householder 1941, der gezeigt hat, dass bei anderen Prosaschriftstellern der Kaiserzeit eine ähnliche Präferenz von Euripides herrscht. Zur Technik der Parodie bei Lukian s.u. S. $186 \mathrm{f}$.

7 Dabei ist die genaue zeitliche Eingrenzung der Zweiten Sophistik ebenso umstritten wie die Bestimmung ihrer Charakteristika; vgl. zur Diskussion u. a. Swain 1996, 1-7 und Whitmarsh 2005. Zur Bedeutung der Bildung in diesem Zeitraum siehe Schmitz 1997 und Borg 2004.

8 Vgl. hierzu ausführlich Baumbach u.v. Möllendorff 2017, 59-99. 
dessen Werke (wie der Büchernarr) durch lautes Lesen bzw. Vorlesen ${ }^{9} \mathrm{zu}$ rezipieren bzw. rezitieren, sondern man muss dies ebenso in Kenntnis der historischen bzw. literarischen Entstehungs- und Wirkungskontexte tun wie mit Blick auf die aktuellen Adressaten. Für eine wirksame Vermittlung der literarischen Tradition soll man gewissermaßen in die Rolle eines Boten einer Tragödie schlüpfen und das Geschehene (= die Tradition) überzeugend vermitteln - und genau in dieser Rolle ist der ungelehrte Büchernarr gescheitert, da er das im Botenbericht der Bakchen geschilderte Zerreißen des König Pentheus durch seine Mutter Agaue (vgl. Eur. Ba. 1043-1152) in offenbar so schlechter Performanz (Gestik, Mimik, Intonation) dargeboten hat, dass Demetrios sich aus Mitleid mit Pentheus gezwungen sah, den sparagmós von der Handlungsebene der Tragödie auf ihr Speichermedium, das Buch des ungelehrten Büchernarren, $\mathrm{zu}$ übertragen.

Die Anekdote enthält keinerlei Hinweise darauf, wie man Euripides' Werke angemessen lesen bzw. rezitieren sollte, sondern legt den Fokus auf die dramatische Wirkkraft, die Euripides' Tragödien in Lukians Euvre entfalten: ,Begegnungen“ mit Euripides im wörtlichen wie im übertragenen Sinn aktivieren die innerfiktiven Rezipienten zu eigenen (dramatischen) Handlungen, die sowohl das Gemachtsein (poiesis) einzelner Schriften prägen als auch poetologische Reflexionen über Tradition und Innovation im literarischen Schaffen Lukians begleiten. Dabei fällt auf, dass auf der Figurenebene häufig ein Scheitern der Euripides-Rezeptionen zu beobachten ist: Wie im Adversus Indoctum fällt ,Euripides‘ in Lukians Schriften in solchen Fällen entweder in die Hände von Leuten, die sein Potential nicht nutzen (können) bzw. durch eine als unpassend empfundene Rezeptionsweise von anderen Figuren in ihrer Unbildung entlarvt werden, oder die Euripidesrezeptionen können - wenn sie von Gebildeten stammen - ihre Wirkungsintentionen auf der Figurenebene nicht entfalten. In beiden Fällen geht es Lukian nicht um eine Abwendung von Euripides, geschweige denn um eine grundsätzliche Kritik an der durch Euripides repräsentierten literarischen Tradition, die von vielen Autoren der Zweiten Sophistik als ,klassisch“ empfunden und - nicht zuletzt von Lukian selbst - sprachlich-stilistisch nachgeahmt wurde, sondern um einen neuen Umgang mit den kanonischen Autoren, der durch die Abgrenzung von unpassenden (ungebildeten) oder ,traditionellen' (gebildeten) Rezeptionsformen seiner Tragödien für die impliziten Rezipienten von Lukians Schriften sichtbar wird. Man kann daher von einem produktiven Scheitern der Euripides-Rezeptionen sprechen, insofern ein Rezipient angehalten ist, nicht nur nach den Gründen für das Scheitern, sondern auch nach möglichen Funktionen und Wirkungsabsichten der inszenierten Euripides-Rezeptionen zu fragen, die sich über die Handlungsebene hinaus im Dialog zwischen Text und Rezipienten entfalten und auf das Gemachtsein der Werke selbst erstrecken.

Für eine solche metapoetische Funktionalisierung von Euripides spricht rein formal bereits die Beobachtung, dass vier auktorial konnotierten Sprecherfiguren

9 Zur Diskussion vgl. Balogh 1927, Vogt-Spira 1991, Gavrilrov 1997, Burfeind 2002 und Busch 2002. 
Lukians - Menipp, Lykinos, Parrhesiades und der Syrer ${ }^{10}$ - Euripides-Zitate im Munde führen und dass in Lukians wichtigster literarischer Neuschöpfung, dem Komödischen Dialog, ${ }^{11}$ mehrfach an prominenter Stelle auf Euripides Bezug genommen wird. Dass speziell Werke des Euripides eine solche Funktion erhalten, kann mit der hohen Popularität und besonderen Wirkkraft seiner Werke begründet werden, die Lukian zu Beginn seiner Schrift über die Art und Weise, „wie man Geschichte schreiben soll“ (Quomodo historia conscribenda sit) mit der Anekdote einer einzigartigen EuripidesManie, die die Bürger von Abdera nach einer Aufführung der Andromache befällt und passive Theaterbesucher zu aktiven ,Schauspielern' macht, unterstreicht:

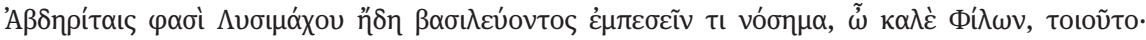

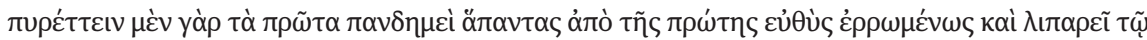

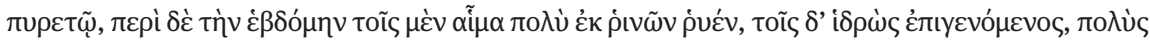

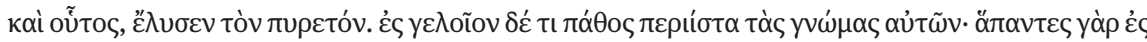

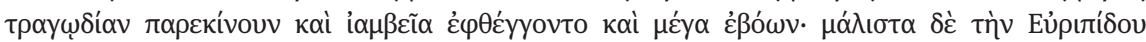

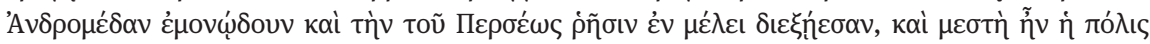

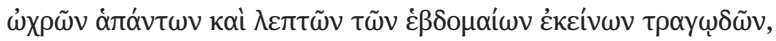

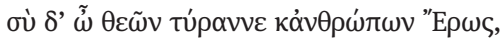

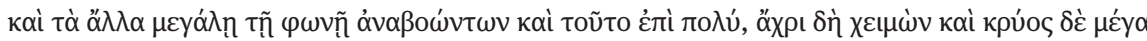

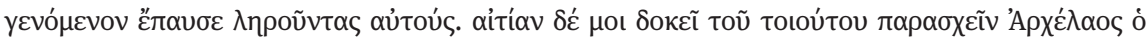

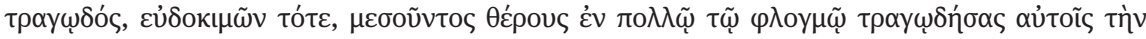

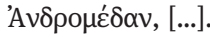

Die Abderiten - so wird berichtet, mein lieber Philon - hat zur Regierungszeit des Königs Lysimachos eine Krankheit von besonderer Art befallen: Zuerst erkrankten alle an einem Fieber, das gleich vom ersten Tag an heftig und anhaltend war; um den siebenten Tag setzte dann bei den einen starkes Nasenbluten ein, bei anderen erfolgte reichlicher Schweißausbruch und daraufhin ging das Fieber herunter. Ganz komisch hat sich aber die Krankheit auf ihren Geisteszustand ausgewirkt: Alle waren nämlich wie verrückt auf die Tragödie und deklamierten in Jamben und zwar in höchster Lautstärke; hauptsächlich trugen sie die Solo-Arien der Andromeda des Euripides vor und rezitierten abwechselnd Teile aus dem Monolog des Perseus; die ganze Stadt war voll von diesen bleichen und abgezehrten Siebentags-Tragöden, die

„Du, über Götter und Menschen grausamer Herrscher, Eros“ [Eur. fr. 136,1 Kannicht] ${ }^{12}$

und so fort mit lauter Stimme brüllten, und das lange Zeit, bis der Winter und ein starker Frost einsetzten und ihrem Geplärr ein Ende machten. Schuld an alledem war, glaube ich, der damals berühmte tragische Schauspieler Archelaos, der mitten im Sommer bei glühender Hitze ihnen die Andromeda vorgespielt hatte, [...]. (Hist. consc. 1,1-22, Übs. Homeyer)

Die Anekdote, die einen Hinweis auf ganzjährige Aufführungen von Dramen in hellenistischer Zeit ${ }^{13}$ gibt, könnte als ,Beleg' für die pathologische Wirkkraft ${ }^{14}$ und Po-

10 Hierzu vgl. Baumbach u.v. Möllendorff 2017, 13-57.

11 Zum Begriff und Konzept des Komödischen Dialogs vgl. Baumbach u.v. Möllendorff 2017, 176 - 209.

12 Zur Überlieferung und unterschiedlichen Gestaltung des Verses bei Athenaios und Lukian vgl. Klimek-Winter 1993, 245-9.

13 Siehe auch den Beitrag von Martin Hose in diesem Band, S. 34f. Die ,Datierung“ der Anekdote erfolgt über den Hinweis auf, die Tage des [Diadochenkönigs] Lysimachos‘. 
pularität des Euripides verstanden werden, den jeder Depp - und als solche galten die Abderiten nicht erst seit Ciceros sprichwörtlichem Ausruf „Hic Abdera“ (Att. 4,16,6) auswendig lernen und (re)zitieren konnte. Eine weitere Bedeutungsebene eröffnet sich durch den Bezug der Anekdote auf das ,Historiographiefieber', das - wie der Erzähler berichtet - nach den römischen Erfolgen in den Partherkriegen (161-166 n. Chr.) unter zeitgenössischen ,Gebildeten' (Hist. conscr. 2) ausgebrochen war und zu zahllosen Abhandlungen geführt hatte, die in Quomodo historia conscribenda sit aufs Korn genommen werden. In beiden Fällen wird das epidemische Übermaß der Präsentation eines Werkes (Euripides’ Andromeda) bzw. Themas (Partherkriege) betont, wobei sich die Kritik an der Euripides-,Epidemie‘ zunächst auf das Zuviel an Euripides

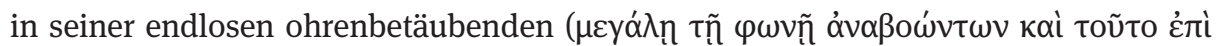
$\pi о \lambda u ́)$ rezitierenden Wiederholung bezieht und später über die Parallelisierung der Abderiten mit den - im weiteren Verlauf des Werkes als „unfähig“ bzw. „schlecht“ (Hist. conscr. 7) entlarvten - ,Historiographen', die sich bei ihren Darstellungen auf die unreflektierte Nachahmung von klassischen Gattungsvorbildern beschränkten, auf die Rezitierenden selbst zurückwirkt. Wie im Adversus Indoctum werden die Schreihälse in ihrem unpassenden Tun am Ende zum Schweigen gebracht, wobei sich innerhalb der Anekdote eine Art Katharsis vollzieht, die sich auf die Wirkungsintention der ganzen Schrift übertragen lässt: So wie der Winter den erhitzten Gemütern der Abderiten die reinigende Abkühlung (= das Ende ihres Tragödienspiels) bringt, so wird ,Lukian“ den Geschichtsschreibern der Partherkriege mit seiner Schrift ihre Grenzen aufzeigen und die Historiographie-Manie stoppen. Genau dafür ist der ,euripideische“ Inhalt gut gewählt, da er eine weitere Parallelisierung ermöglicht: Indem die Abderiten Passagen aus dem Monolog des Perseus rezitieren (und diesen als Sprecher verkörpern), stehen zu Beginn von Quomodo historia conscribenda sit viele Perseus-Figuren als Bekämpfer eines Seeungeheuers auf der imaginären Bühne, die sich für einen Lukian-Rezipienten bei der Lektüre der Anekdote öffnet. Nach ihrem Abtritt betritt eine Figur als Bekämpfer vieler Historiographen die Bühne der Narration, in dessen späterem Rat an die Geschichtsschreiber, sich bei ihren Darstellungen (wie er) von einem poetischen, auf einem Pferd reitenden Geist (45) beflügeln zu lassen, das Bild des auf Bellerophon fliegenden Perseus zumindest mitschwingt. Damit verleiht der Euripides-Bezug in der Abderiten-Anekdote Lukians literaturkritischer Abhandlung über die Art und Weise, ,Geschichte zu schreiben', nicht nur eine dramatisch-poetische Note, sondern verbindet mit der kreative Verbindung von Tragödie und Geschichtsschreibung in dem einzigen aus der Antike erhaltenen Traktat über die Historiographie möglicherweise einen Hinweis auf die literarische Tradition der sog. ,tragischen' Geschichtsschreibung. ${ }^{15}$

14 Vgl. Bing 2011, 5. Zur Deutung der Anekdote und zu ihren möglichen literarischen Vorläufern vgl. Porod 2013, 257-266 und v. Möllendorff 2001, der die Anekdote als Enthusiasmos-Parodie deutet. 15 Zur Diskussion über die Theorie und Praxis einer tragischen Geschichtsschreibung vgl. Porod 2013, 133-152. 
Doch nicht nur Ungebildete wie der ungelehrte Büchernarr oder die Abderiten scheitern in Lukians Werken mit bzw. an ihren Euripides-Rezeptionen, sondern auch Gebildete: Ein prominentes Beispiel findet sich im Piscator, in dem der ,Freiredner Parrhesiades wegen Verunglimpfung der Philosophenschulen angeklagt wird, sich jedoch als Gebildeter erweist und erfolgreich verteidigen kann. Zu Beginn der Auseinandersetzung sieht es jedoch schlecht für Parrhesiades aus, da er den ersten, mit Homer-Zitaten geführten Wortwechsel mit dem anklagenden Platon verliert und sein Heil bei Euripides suchen muss:

\section{ПАРPH $Г I A \triangle H \Sigma$}

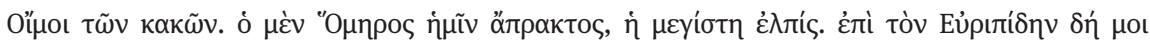

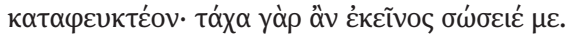

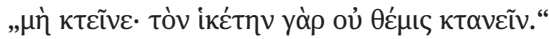

$\Pi \Lambda A T \Omega N$

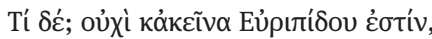

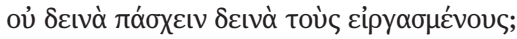

\section{Parrhesiades:}

O welch Unglück! Homer, der meine größte Hoffnung war, ist wirkungslos; jetzt muss ich Zuflucht zu Euripides nehmen, vielleicht wird jener mich retten:

„Töte mich nicht! Denn es ist Unrecht, den Schutzflehenden zu töten.“

\section{Platon:}

Was? Stammt nicht auch dies von Euripides:

„Nicht ist es schlimm zu leiden für die, die Schlimmes taten“? (Pisc. 3)

Parrhesiades' Rettungsanker - ein Euripides-Zitat aus einer uns unbekannten Tragödie (fr. 937 Kannicht) - hat nicht die gewünschte Wirkung, da Platon mit einem Vers aus dem Orestes (413) das poetisch eingeforderte ,Recht auf Verschonung“ zurückweisen kann. Dadurch, dass Platon Parrhesiades zweimal mit seinen eigenen Waffen schlagen kann und der Disput mit geliehenen Worten sich totläuft, liegt der Fokus der Dichterrezeptionen auf ihrem Scheitern: Was als scheinbar auf höchstem Niveau geführter Disput zweier Gebildeter mit und über griechische Bildung begann, ${ }^{16}$ endet in einem Fiasko: Parrhesiades' Vereinnahmung einer dichterischen Autorität wird als einseitig und verkürzend entlarvt, da jeder Gebildete, der (wie Platon) Euripides gelesen hat, natürlich ebenfalls Euripides-Stellen, die als Gegenargument verwendet werden können, kennt. Zudem wird am Beispiel von Euripides und Homer deutlich, dass die dichterische literarische Tradition keine überzeitliche moralische Instanz ist, auf die man sich berufen kann - eine Erfahrung, die im lukianischen Euvre auch Timokles machen muss, dessen ,euripideischen` Gottesbeweis im Juppiter Tragoedus (41) der Epikureer Damis mit zwei Euripides-Zitaten (fr. 480 u. fr. 941 Kannicht) widerlegt, in dem die Existenz der Götter bezweifelt wird. Vor diesem Hintergrund scheint eine Wirkungsabsicht der Euripides-Zitate im Piscator und Juppiter Tragoedus

16 Dabei wird die zeitlose Bedeutung des Euripides unterstrichen, da dieser unter Gebildeten zu Platons Lebzeiten ebenso bekannt ist wie im 2. Jh. Lukians, in dem der Piscator spielt. 
das Hinterfragen eines Bildungshabitus zu sein, der sich auf literarische Traditionen bzw. Autoritäten beruft und sich durch Zitate zu legitimieren sucht, die zu bloßen Systemreferenzen an eine bestimmte Bildung geworden sind. Ein solcher Umgang mit der literarischen Tradition ist weder innovativ noch erfolgreich, was sowohl Platon als auch Parrhesiades zu erkennen scheinen, wenn sie in der Folge zur attischen Prosa zurückkehren und sich in dieser Form auf eine friedliche Lösung des Streites einigen können.

\section{Poetologische Untertöne: Die Aktivierung der Gelehrsamkeit der Rezipienten}

Eine mit dem Juppiter Tragoedus und dem Piscator vergleichbare Kommunikationssituation, in der Euripides eine zentrale Rolle einnimmt, findet sich zu Beginn der Necyomantia, der Unterweltsfahrt Menipps:

MENIPPOE

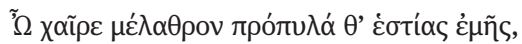
ФI $О \Sigma$

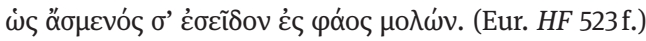

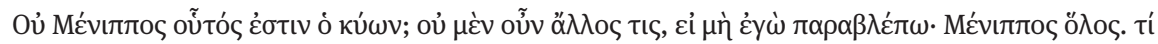

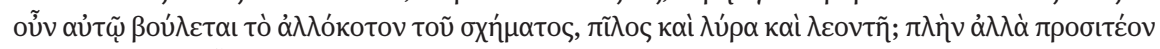

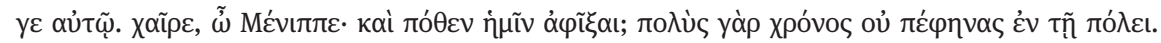
MENIPPOS

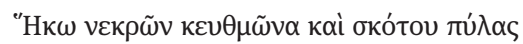

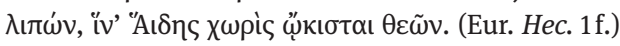

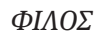

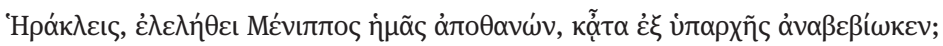
MENIPPOE

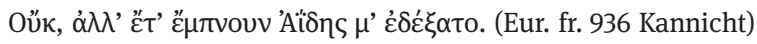

Menipp:

„Seid mir gegrüßt, mein Haus, mein Herd!

Wie freu’ ich mich nach meiner Rückkehr ans Licht, Dich zu sehen!“

Freund:

Ist das nicht Menipp, der Hund? Wahrlich, kein anderer, wenn ich mich nicht täusche. Ganz und gar Menipp! Aber was hat sein seltsamer Aufzug zu bedeuten: Kappe, Leier und Löwenfell? Wie dem auch sei, ich muss zu ihm gehen. Sei gegrüßt, Menipp! Woher des Wegs? Schon lange hast du dich nicht mehr in der Stadt blicken lassen.

Menipp:

„Ich komme von der Totengruft und den Toren der Dunkelheit,

von dort, wo Hades fernab von den Göttern wohnt.“

Freund:

Beim Herakles! Ist Menipp etwa gestorben, ohne dass ich davon wusste, und nun wieder ins Leben zurückgekehrt?

Menipp:

„Keineswegs! Lebend nahm mich Hades auf.“ (Nec. 1, Übs. Baumbach u.v. Möllendorff). 
Folgt man der Logik der Darstellung, dann sind die Verse nicht als Zitate zu verstehen, die Menipp absichtlich verwendet, um als Gebildeter in einen intertextuellen Dialog mit der literarischen Tradition zu treten, sondern eine Reminiszenz an seine Unterhaltung mit Euripides in der Unterwelt, von der inspiriert er tragische Verse spricht. Im Vergleich mit den Wortgefechten im Juppiter Tragoedus und Piscator fällt zunächst auf, dass es keine Hinweise auf die Provenienz der Verse gibt. Damit besteht ein Reiz der Passage in der Aktivierung der textimmanenten und textexternen Rezipienten und ihrer Bildung. Eine solche implizite Aufforderung zur Mitarbeit bei der Recodierung von Bildungselementen durch unmarkierte Intertextualität gehört seit den Anfängen griechischer Dichtung zum Repertoire und wird von Lukian in vielen seiner Werke mit ganz unterschiedlichen Autoren verwendet. Besonders prägnant sind diesbezüglich die Verae Historiae, in denen die textexternen Rezipienten in der Vorrede explizit dazu aufgefordert werden, die folgende anspielungsreiche Reise durch die ,Lügen“ der Literatur vermittels ihrer Bildung mitzuverfolgen und sich (im Erfolgsfall) als Gebildete bestätigt zu sehen:

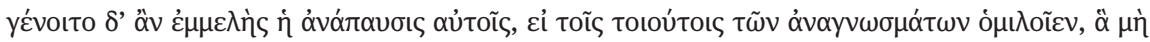

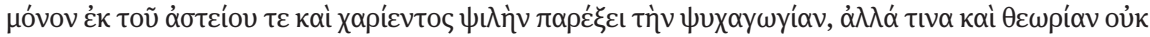

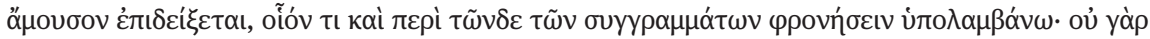

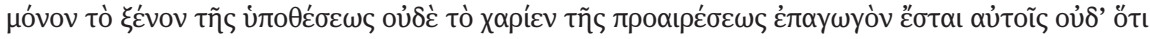

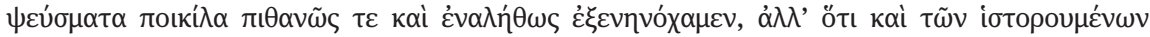

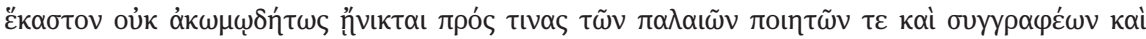

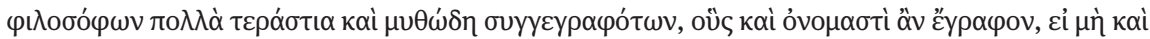

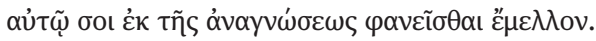

Für sie [= die Leser] dürfte es wohl einen angemessenen Ausgleich darstellen, sich mit Werken zu beschäftigen, die zum einen auf geistreiche und witzige Weise Vergnügen bereiten, zum andern aber auch eine anspruchsvolle Betrachtung ermöglichen; und ich hoffe, dass sie über die vorliegenden Bücher ebenso denken. Denn nicht nur das ungewöhnliche Thema und sein unterhaltsamer Zweck sowie die zahlreichen Lügen, die ich glaubwürdig vorgebracht habe, werden sie anlocken, sondern vor allem die Tatsache, dass jedes einzelne Stück meiner Geschichte nicht unwitzige Anspielungen auf alte Dichter, Geschichtsschreiber und Philosophen enthält, die ihrerseits viel Wundersames und Fabelhaftes geschrieben haben: Personen, die ich auch namentlich erwähnt hätte, wenn man sie sich bei der Lektüre nicht von selbst erschließen könnte. (VH $1,2)$

Im Fall der Necyomantia zeigt sich jedoch schnell, dass diese Erwartungshaltung nicht erfüllt wird: Zum einen ist auf der Figurenebene der Freund nicht in der Lage, die Verse zuzuordnen - zwar erkennt er, dass es sich um Tragödienverse handelt ( $\pi \alpha \tilde{\sigma} \sigma \alpha \mathrm{l}, \mu \alpha-$

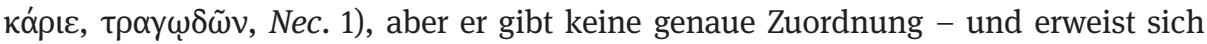
somit als nicht besonders gebildet; auch das Unvermögen, die Verkleidungen den drei Unterweltsfahrern zuzuordnen (Kappe = Odysseus, Leier = Orpheus, Löwenfell = Herakles) sowie sein Glaube an Wiederauferstehung bauen eher Distanz als Nähe zu einem gebildeten Rezipientenpublikum der Necyomantia auf. Demgegenüber könnte ein gebildeter Leser die Stellen bereits als euripideisch erkannt haben, allerdings wird 
die unmarkierte Intertextualität wenig später in eine markierte überführt, da Menipp selbst Euripides als seine Quelle nennt (Nec. 1).

Mit Blick auf den mit Euripides (und Homer) geführten Bildungsdiskurs wird auf der Figurenebene erneut ein Scheitern vorgestellt, da erst nach der Abkehr vom poetischen Sprechen eine Verständigung zwischen den Figuren möglich ist. Und ähnlich wie im Juppiter Tragoedus und im Piscator erscheint auch in der Necyomantia für einen textexternen Rezipienten der interpretative Mehrwert einer möglichen intertextuellen Rückbindung der Zitate an ihre Kontexte (Hercules Furens und Hecuba) gering. ${ }^{17}$ Gleichwohl ergibt sich eine Funktion der Euripides-Zitate, wenn man die durch sie gemachten Referenzen an die Schreibweise der so genannten ,menippeischen Satire' betrachtet, die auf den Kyniker Menipp von Gadara zurückgeht, dessen heute verlorenen Schriften aus dem 3. Jh. v. Chr. Lukian gekannt hat und deren formale

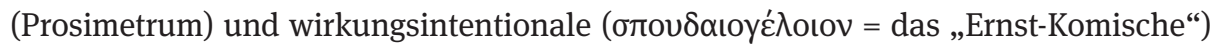
Charakteristika mehrfach bei der poetologischen Reflexion über das eigene literarische Werk erwähnt werden. So erhebt Diogenes im Piscator gegen die auktorial konnotierte Sprecherfigur Parrhesiades den Vorwurf, er habe „Menipp, unseren

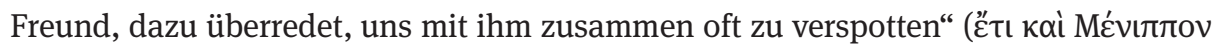

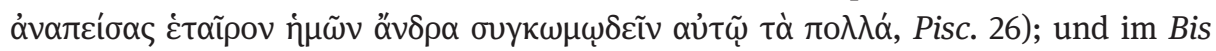
Accusatus, in der ,Lukian“ wegen seinen literarischen Neuschöpfungen angeklagt wird, beklagt sich der personifizierte platonische Dialog bitter über die Zumischung von Versen, die er aus der Feder eines ,Syrers` (= Lukian) erlitten habe:

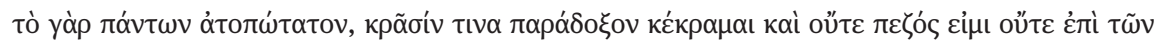

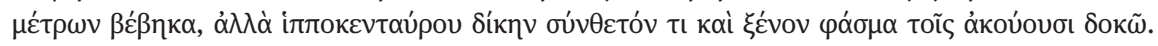

Das Ungehörigste von allem aber ist, dass ich zu einem unglaublichen Mischmasch verunstaltet wurde und weder zu Fuß gehe noch auf Versen schreite, sondern meinen Zuhörern als ein Mischwesen nach Art eines Hippokentauren und als fremdartiges Gebilde erscheine. (Bis Acc. 33, Übs. Baumbach u. v. Möllendorff)

Vor diesem Hintergrund liegt es nahe, den Beginn der Necyomantia als eine Referenz an die ,menippeische Satire' zu verstehen, zumal Menipp dort selbst als Sprecher auftritt. Auffallend ist jedoch, dass unmittelbar auf das Sich-Einschreiben in die Menippea eine formale Abwendung von eben dieser erfolgt, da der Rest der Necyomantia - abgesehen von vier kurzen Homer-Zitaten (Nec. 9, 10, 21) - in Prosa erzählt wird. Ein ähnlicher Befund ergibt sich mit Blick auf den Piscator und den Juppiter Tragoedus. Leider ist die Überlieferung des vermeintlichen Begründers dieser literarischen Form, des Kynikers Menipp von Gadara, zu fragmentarisch, um ein klares Bild

17 Zudem ist jeweils zu prüfen, ob es sich bei einem zitierten Vers ggf. um ein sprichwörtlich verwendetes Zitat handelt, das entsprechend häufig bei anderen Autoren belegt oder in Anthologien zu finden war; in solchen Fällen schwächt sich die intertextuelle Spur gegenüber einer allgemeinen, vom Werkkontext gelösten Verwendungsweise ab; vgl. hierzu auch die Analysen der Euripides-Zitate von Karavas 2005, 139-160. 
von den Anfängen der Menippea zu geben. Aber die für Menipp bezeugte Mischung von Prosa und Vers in seinen verlorenen Werken könnte in einer ähnlichen Weise mit Dichterzitaten erfolgt sein, wie Lukian sie in der Necyomantia anklingen lässt, sie wäre dort aber wahrscheinlich konsequenter ausgeführt gewesen. Nimmt man die Wirkungsintention des „Ernst-Komischen“ hinzu, die Menipps Satiren ausgezeichnet haben soll, dann wird die Verwendung des Euripides deutlich, die Lukian mit seiner

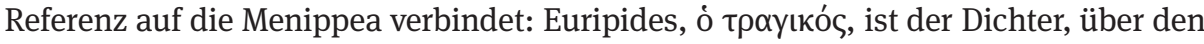
am besten ,ernste، Themen eingespielt und ,ernste‘ Wirkungsabsichten erzeugt werden konnten. Die gemachten Beobachtungen zu den drei besprochenen Werken lassen den Schluss zu, dass Lukian durch die verwendeten Euripides-Zitate und die Referenzen an die menippeische Satire bestimmte literarische Traditionen (Menippea) und Rezeptionsweisen (von Euripides) aufruft, um sie zu verlassen: Das Prosimetrum wird nicht weiterverfolgt, die Euripides-Zitate laufen ins Leere.

Damit - und das ist Teil der Wirkungsabsicht der lukianischen Verfahrensweise drängt sich die Frage nach einem neuen Umgang mit der literarischen Tradition und dem Zitieren von literarischen Autoritäten auf. Wie könnte dieser aussehen?

\section{Produktives Scheitern: Euripides-Parodie und literarische Hybridität}

In den besprochenen Texten finden sich neben dem Aufrufen bestimmter literarischer Werke über Zitate parodistische Verwendungen. Diese Technik scheint Lukian besonders wichtig zu sein, da sie nicht nur in vielen Werken vorkommt, sondern dreimal explizit als solche bezeichnet wird (Juppiter Tragoedus 14, Apologia 10, Contemplantes 14), am pointiertesten am Beispiel von Euripides: In der Apologie $e^{18}$ leiht sich der IchErzähler bei seiner Verteidigung gegen den Vorwurf, gegen seine eigenen in der Schrift De mercede conductis vorgebrachten Bedenken ${ }^{19}$ nun selbst einen bezahlten Job im Establishment angenommen zu haben, Worte der Medea:

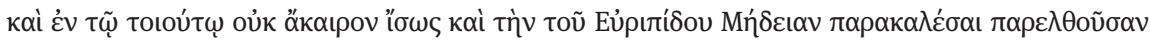

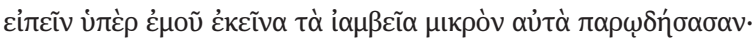

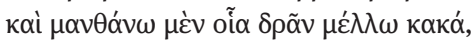

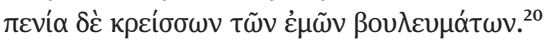

In solch einer Situation ist es vielleicht nicht unpassend, die Medea des Euripides als Beistand herbeizurufen, damit sie zu meiner Verteidigung folgende leicht parodierten Jamben spricht:

18 Zur autobiographischen Pose und zur literarischen Tradition vgl. Hafner 2017, 17-36.

19 Das subversive Spiel mit der ,eigenen‘ Autorität diskutiert Whitmarsh 2001, $292 \mathrm{f}$.

20 Die Parodie legt die Übersetzung „vernünftige Entscheidungen“ nahe, die für die Euripides-Stelle selbst umstritten ist - vgl. zur Diskussion Gill 1996, 216 - 218, Mastronarde 2002, 345 sowie Hafner 2017, $106-108$. 
Ich weiß, welch schlimme Dinge ich zu tun gedenke, aber die Armut ist stärker als meine Pläne. (Apol. 10)

Das Zitat stammt aus Euripides’ Medea (1078f.), allerdings steht bei Euripides in Vers

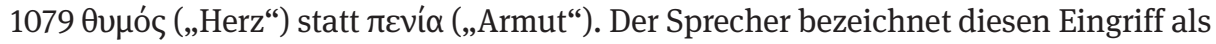

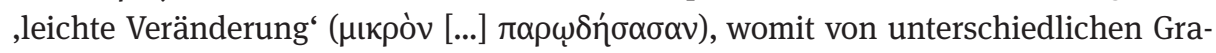
den der ,Parodie‘ ausgegangen wird. Dieses Verfahren bedeutet für die gebildeten Rezipienten Lukians eine Aufforderung zur Mitarbeit, da sie sowohl die parodistische Veränderung erkennen als auch den jeweiligen Kontext, aus dem das Zitat stammt, präsent haben müssen, um den Sinn der Parodie und ihre Wirkungsabsicht zu erkennen. ${ }^{21}$ Im parodistischen Sprechakt findet eine besondere Form der Aneignung euripideischer Sprache statt, die zu erkennen eine besondere und nicht mehr nur eine allgemeine Euripides-Bildung erfordert. Und eben diese reizvolle parodistische Verfremdung kann skalierend eingesetzt werden: Man kann wie an dieser Stelle nur ein Wort ändern ( $\mu$ ıрòv [...] $\pi \alpha \rho \omega \delta \eta ́ \sigma \alpha \sigma \alpha v)$ oder mehrere, und man kann die Parodie sowohl durch eigene Hinzudichtungen erzeugen als auch durch Kompilation von mehreren Texten - zwei Verfahrensweisen, die sich auch in der Poiesis der CentoDichtung finden, die Lukian ebenfalls verwendet. ${ }^{22}$ Ein schönes Beispiel findet sich im Juppiter Tragoedus:

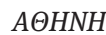

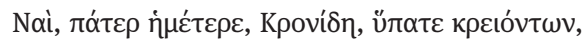

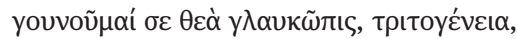

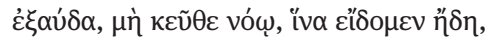

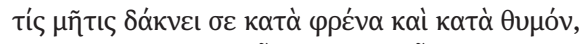

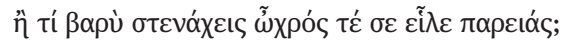
ZEYS

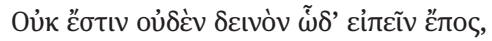

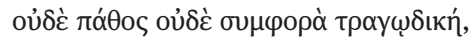

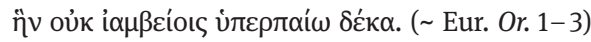

\section{A@HNH}

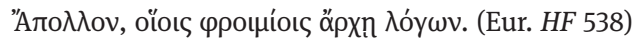

ZEYS

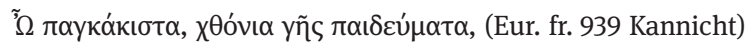

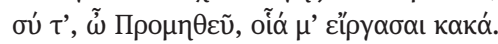

A $\Theta H N H$

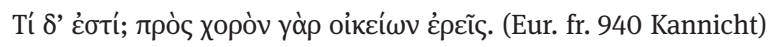

ZEYS

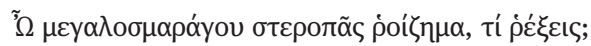

$H P A$

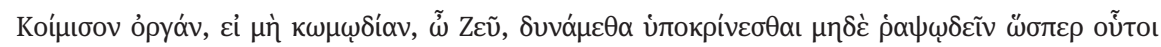

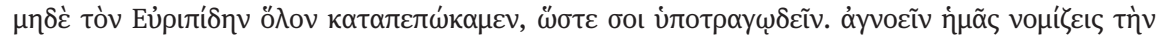

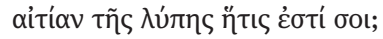

21 Siehe hierzu auch Baumbach u. v. Möllendorff 2017, $211 \mathrm{f}$.

22 Vgl. Baumbach u. v. Möllendorff 2017, 209-216. 


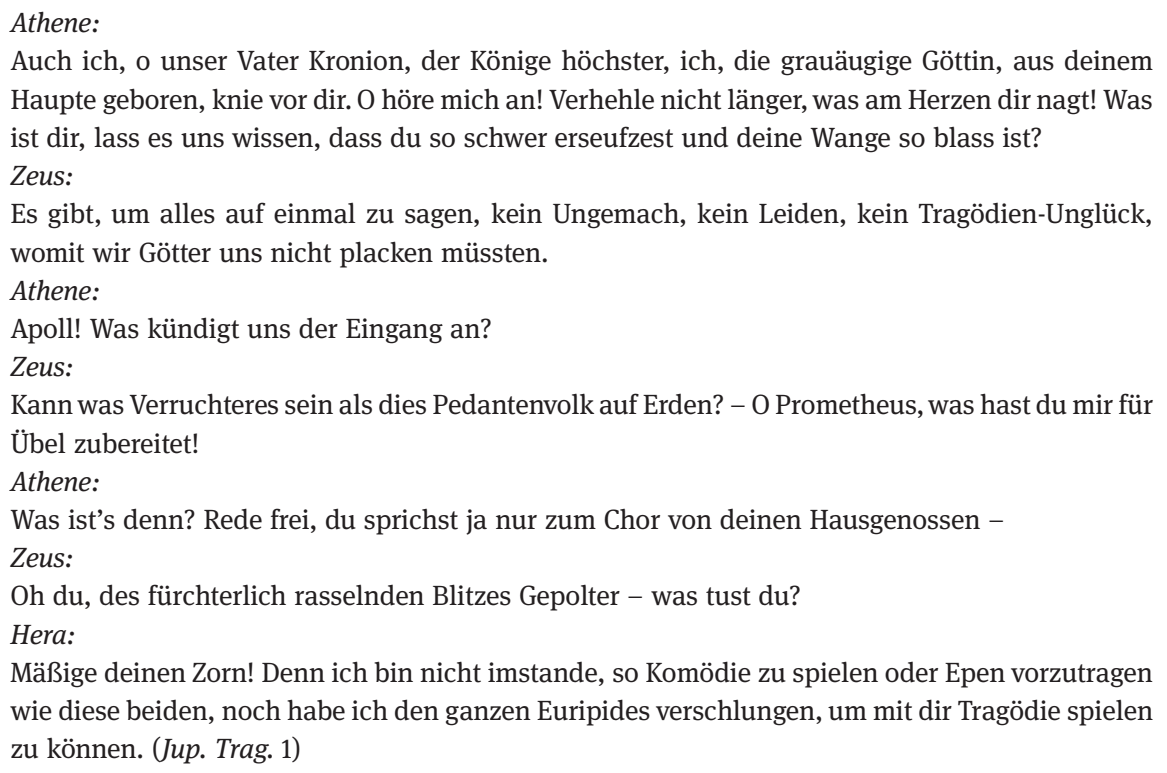

Der besondere Reiz für die Rezipienten der Schrift liegt zunächst darin, dass es keinerlei Markierungen der intertextuellen Bezüge gibt und im Unterschied zur Necyomantia auch keine spätere Auflösung ihrer Herkunft. Damit ist ein Rezipient von Beginn an gefordert, mit Hilfe seiner Bildung die Verse ihren ursprünglichen Kontexten zuzuordnen. Alarmiert vom schwindenden Götterglauben der Menschen diskutieren die Götter auf dem Olymp ihr weiteres Vorgehen, bevor sie im zweiten Teil des Dialogs dem bereits erwähnten Streitgespräch zwischen Timokles und Damis lauschen. Inspiriert von der einleitenden Homer-Parodie Athenes parodiert Zeus die ersten Verse des euripideischen Orestes, die dort Elektra spricht:

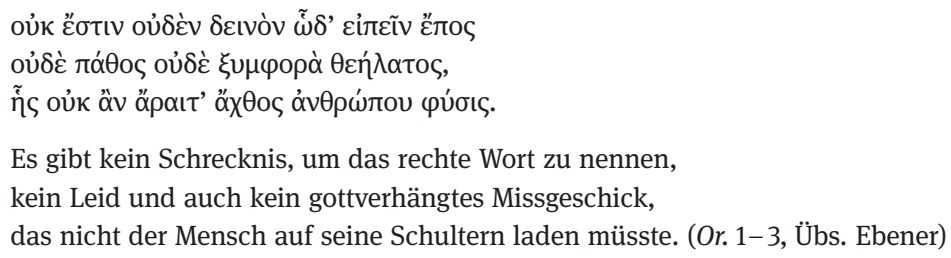

Inhaltlich wird mit dem Anfang des Orestes die ganze Tragödie des Euripides aufgerufen, figürlich wird eine verzweifelte, ihren Untergang erwartende Elektra auf die lukianische Bühne des Juppiter Tragoedus geholt. Damit ist Zeus - wie auch der Titel andeutet - zu einem zweiten Euripides geworden, der als Dichter ebenso produktiv tätig ist wie er als Figur in der Rezeption der Menschen auf ,tragische“ Weise behandelt wird. Die Parodie verändert auf der Inhaltsebene das Abhängigkeitsverhältnis von Mensch und Gott und reflektiert das mit einem ,tragischen' Schicksal verbundene poetische Schaffen: Lukians Zeus übertrifft jedes tragisches Schicksal - und damit 
auch jedes in einer Tragödie von Euripides geschilderte Schicksal - weshalb poetologisch gelesen, eine neue Schreibweise angemessen ist, diese Besonderheit adäquat auszudrücken - eben die Parodie.

Athene antwortet mit einem Vers aus dem euripideischen Hercules Furens, den Herakles bei seiner Rückkehr nach Theben zu seiner verzweifelten Frau Megara spricht, die um ihr Leben und das seiner Kinder fürchtet. Die Situation für Zeus ist also doppelt tragisch: Nicht nur seine Existenz, sondern auch die seiner Familie (= der Götter, die ja fast alle von ihm abstammen) ist bedroht. Sein zweites Euripides-Zitat mit der Verwünschung der Prometheus-Kinder - evtl. wieder eine Parodie, wir haben nur das Fragment (fr. 939 Kannicht) - kann von Hera falsch verstanden werden, da Prometheus nicht nur den Menschen, sondern speziell auch die Frau geschaffen hat, die sich als solche angesprochen fühlen mag. Textimmanente Figuren werden so ebenso wie die textexternen Rezipienten in ein kompliziertes euripideisch-lukianisches Drama versetzt, das mit den euripideischen Kontexten arbeitet und über die Parodie neue dramatische Situationen bzw. Konstellationen entstehen lässt.

Und wenn Zeus in der Folge weiter parodiert und Hermes, der zu Beginn des Juppiter Tragoedus eine Menander-Parodie und damit ein dezidiert komisches Element in das Stück eingebracht hatte, Zeus später zu einer Rede im Stil des Demosthenes rät (14), zu der Zeus dann auch - leicht parodierend - anhebt, dann liegt hier eine neue Art des Dialogs vor, der in der Euripides-Parodie seinen Ausgangspunkt hat und in seinen unterschiedlichen Bestandteilen genau den hybriden literarischen Gebilden entspricht, die Lukian in kreativer Auseinandersetzung mit der literarischen Tradition entstehen lässt. Dazu gehört in erster Linie der Komödische Dialog, den Lukian in drei Werken (Bis Accusatus, Piscator, Prometheus es in verbis) mit unterschiedlichen Nuancen über die Vermischung von verschiedenen Gattungen (philosophischer Dialog, Komödie) definiert und dem er neben einer komischen Wirkungsabsicht eine starke Handlungsorientiertheit sowie eine philosophische Thematik $^{23}$ zuschreibt. Weitere literarische Mischwesen kommen hinzu, die Lukian ohne spezifische Definition als Hippokentauren oder Kentauren bezeichnen würde, folgt man den Überlegungen im Prometheus es in verbis. Dort fügt der anonyme Sprecher, nachdem er zunächst speziell von der Form des Komödischen Dialog gesprochen hat, Überlegungen zur Hybridität von Werken hinzu, die ein Prinzip erkennen lassen, das in vielen lukianischen Werken zur Anwendung kommt:

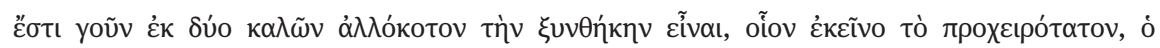

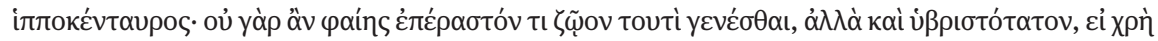

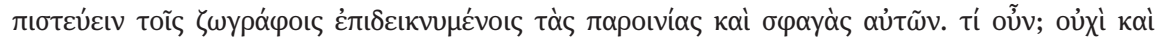

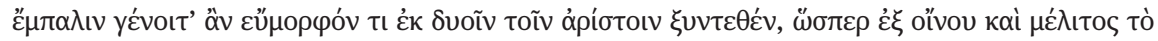

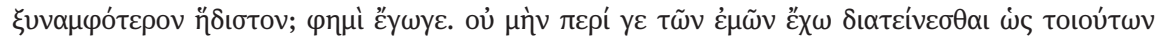

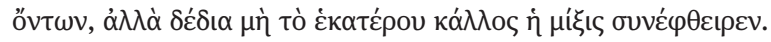

23 Vgl. Baumbach u. v. Möllendorff 2017, 176-209. 
Es kann vorkommen, dass die Zusammenstellung von zwei schönen Bestandteilen scheußlich ist. Nehmen wir etwa das naheliegende Beispiel des Kentauren: Den würdest du ja wohl kaum als reizvolles Wesen bezeichnen - vielmehr verletzt er jedes Maß, wenn man den Malern Glauben schenken darf, die die alkoholischen Exzesse und die blutigen Auseinandersetzungen dieser Wesen darstellen. Aber was denn! Könnte nicht auch andersherum, wenn man zwei erstklassige Bestandteile miteinander kombiniert, ein geschmackvolles Ergebnis dabei herauskommen, so wie die köstliche Mischung von Wein und Honig? Das will ich doch meinen! Allerdings zögere ich, Entsprechendes von meinem Werk zu behaupten. Vielmehr befürchte ich, dass die jeweilige Schönheit der beiden Bestandteile durch ihre Mischung verdorben worden ist. (Prom. 5)

Sieht man einmal von der abschließenden captatio benevolentiae ab, mit der der Sprecher auf den sein eigenes Werk prägenden Komödischen Dialog verweist, wird hier eine allgemeine Beschreibung der literarischen Hybridität gegeben, die Lukians Schriften durch das Vermischen von unterschiedlichen Bestandteilen aus der literarischen Tradition charakterisiert. Analog zur Technik der Parodie innerhalb der einzelnen Werke ist es die kreative Zusammensetzung der Werke selbst, die über ein ,generic enrichment' hinausgehend literarische Mischwesen bzw. Kentauren erschafft, für deren ästhetische Beurteilung durch die Rezipienten es keine (generischen) Muster gibt. Mit dieser Poiesis werden vertraute Produktions- und Rezeptionsweisen durchbrochen und innovative Umgangsweisen mit kanonischen Autoren wie Euripides aufgezeigt.

Das für den Umgang mit der Tragödie (und Euripides als ihrem Hauptvertreter) eindrücklichste Beispiel ist die Schrift Podagra: ${ }^{24}$ Ein gichtgeplagter Greis, der über seine Krankheit klagt, wird Zeuge, wie die personifizierte ,Gicht‘ als Gottheit vor ihren Anhängern, einem Chor von gichtgeplagten Männern, erscheint. Nach einer Hasstirade der Göttin gegen die Existenz von Heilmitteln und deren vermeintliche Wirkung kommt ein Bote und berichtet, dass angeblich von syrischen Ärzten ein wirksames Mittel gegen die Gicht erfunden wurde, was sich jedoch zur Freude der Podagra als falsch erweist, da die Ärzte am Ende selbst an Gicht erkranken und ihr Mittel nichts dagegen bewirken kann. Der Text ist von Beginn an durchzogen von Anspielungen und Zitaten aus den Tragikern, von denen die mit Abstand meisten aus Euripides stammen. ${ }^{25}$ An insgesamt 14 Stellen werden Euripides-Verse teilweise oder ganz zitiert, wobei durch die Verwendung von Eur. Iph. Taur. 948 in Vers 1 und die Parodie von vier typischen Schlussversen euripideischer Tragödien (vgl. Alkestis, Helena, Bakchen und z.T. Medea) im abschließenden Chorlied, ein Rahmen gebildet wird, der die Erwartungshaltung an ein euripideisch gestaltetes Stück weckt und diese bis zum Schluss offenhält. Doch nicht nur die Kürze der Podagra (334 Verse), sondern auch ihre un-

24 Die communis opinio hält das Werk seit Zimmermann 1909 für authentisch, vgl. zur Forschungsdiskussion Karavas 2005, 235-242. Vollkommen spekulativ und einer biographistischen Lesart lukianischer Werke geschuldet ist die These, dass Lukian selbst an Gicht gelitten habe - so Macleod 1967, 321: „Furthermore Lucian probably suffered from gout himself. [...] he seems to have had gout himself in his old age.“

25 Vgl. hierzu den Befund von Karavas 2005, 243-299. 
gewöhnliche metrische Gestaltung mit (parodierten) Versen aus der lyrisch-hymnischen Tradition (Pod. 87-111, Anapäste), sotadischen Verse (113-124) und miurischen Hexametern (312-324) verweisen auf den hybriden Charakter der Dichtung, die keiner bestimmten literarischen Vorlage folgt. Falsche Erwartungen weckt auch der gichtgeplagte Greis mit seinem ersten Vers, in dem er mit den Worten des Orestes (Eur. IT

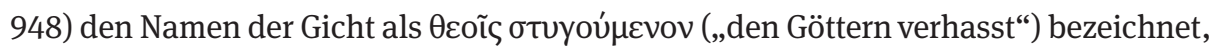
da die Gicht - wie der Verlauf des Stückes zeigen wird - anders als Orestes bei Menschen und Göttern gerade nicht verhasst ist, sondern eine wachsende Zahl an Anhänger besitzt, die im nächsten Moment die ,Bühne‘ betreten und der Göttin ihren Lobpreis singen und zu denen sich auch der Greis selbst zählen wird. Die Möglichkeit der Rekontextualisierung des Ausdrucks in der euripideischen Iphigenie bei den Taurern auf der Rezipientenseite verleiht dem Sprechakt des Greises tragische Ironie, da er nicht weiß, dass die Gicht - analog zu Orestes - am Ende von den Vorwürfen gegen sie freigesprochen und sie unbehelligt, ja ehrenvoll in die menschliche Gemeinschaft aufgenommen werden wird. Zugleich hat der Greis mit dem wörtlichen Sinn seiner Klage in den Augen eines fiktionsexternen Rezipienten Recht - Gicht war in der Antike eine gefürchtete und oft beklagte Krankheit -, so dass die tragische Erwartungshaltung an das Thema ,Gicht' gerade durch die tragische Gestaltung desselben (mit Tragödienversen, die intertextuelle Spuren tragen) in ihr Gegenteil verkehrt wird.

Hier steht die Technik der Parodie von Euripides-Versen im Dienst einer Parodie der durch diese Verse gestalteten Gattung Tragödie selbst, deren sprachliche, metrische, strukturelle und wirkungsästhetische Charakteristika verfremdet werden. ${ }^{26} \mathrm{Am}$ Ende bekommen wir als eine neue literarische Form ein hybrid gestaltetes Miniaturdrama präsentiert, das sein Spiel und seinen Spott nicht nur mit den von Gicht ge-

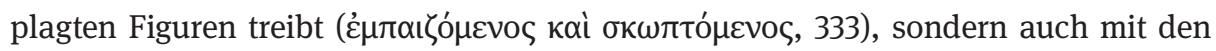
(noch) nicht gichtgeplagten gebildeten Rezipienten, wenn sie in der parodierten euripideischen Exodos das Ende der dramatischen Podagra erwarten: Anders als das Abderitenfieber, das als Anekdote Einmaligkeit für sich reklamiert, ist die Podagra als Krankheit und die Podagra als Text nicht teleologisch angelegt: Ihre Wirkung kommt zu keinem Ende, und genau das ist ihr $\pi \rho \tilde{\alpha} y \mu \alpha$ (334). Als hybride Mischform regt die Podagra zu hybriden Handlungen und Formen an - sowohl intra- wie extradihegetisch: Die syrischen Ärzte mixen in Auseinandersetzung mit ihr Heilmittel aus unterschiedlichsten Dinge zusammen (146-173) und in dem unter Lukians Werken überlieferten Ocypus wird eine weitere Episode aus dem Wirken der Podagra in einer ähnlich hybriden Weise erzählt - natürlich wieder mit Euripides-Versen.

26 Vgl. zu den Elementen der Gattungsparodie die Analyse von Karavas 2005, 321-327. 


\section{Literatur:}

Balogh 1927: József Balogh, „Voces Paginarum. Beiträge zur Geschichte des lauten Lesens und Schreibens“, Philologus 82, 84-109; 202-240.

Baumbach u. v. Möllendorff 2017: Manuel Baumbach u. Peter von Möllendorff, Ein literarischer Prometheus. Lukian von Samosata und die Zweite Sophistik, Heidelberg.

Bing 2011: Peter Bing, „Afterlives of a Tragic Poet: Anecdote, Image and Hypothesis in the Hellenistic Reception of Euripides“, in: Antike \& Abendland 57, 1-17.

Borg 2004: Barbara Borg (Hg.), Paideia: The World of the Second Sophistic, Berlin/New York. Bouquiaux-Simon 1968: Odette Bouquiaux-Simon, Les lectures homériques de Lucien, Brüssel. Burfeind 2002: Carsten Burfeind, „Wen hörte Philippus? Leises Lesen und lautes Vorlesen in der Antike“, Zeitschrift für Neutestamentliche Wissenschaft und die Kunde der Älteren Kirche 93, $138-145$.

Busch 2002: Stephan Busch, „Lautes und leises Lesen in der Antike,“ Rheinisches Museum für Philologie 145, 1- 45.

Gavrilrov 1997: Alexander K. Gavrilov, „Techniques of Reading in Classical Antiquity“, Classical Quarterly 47, 56-73.

Gill 1996: Christopher Gill, Personality in Greek Epic, Tragedy, and Philosophy: The Self in Dialogue, Oxford.

Hafner 2017: Markus Hafner, Lukians Apologie. Eingeleitet, übersetzt und erläutert (Classica Monacensia 50), Tübingen.

Householder 1941: Fred Walter Householder, Literary Quotation and Allusion in Lucian, New York.

Karavas 2005: Orestis Karavas, Lucien et la tragédie (Untersuchungen zur antiken Literatur und Geschichte 76), Berlin/New York.

Klimek-Winter 1993: Rainer Klimek-Winter, Andromedatragödien: Sophokles, Euripides, Livius Andronikos, Ennius, Accius. Text, Einleitung und Kommentar (Beiträge zur Altertumskunde 21), Stuttgart.

Mastronarde 2002: Donald J. Mastronarde, Euripides, Medea, Cambridge.

Macleod 1967: Matthew D. Macleod, Lucian, vol. 8. With an English Translation, Cambridge, Mass./London.

v. Möllendorff 2001: Peter von Möllendorff, „Frigid Enthusiasts: Lucian on Writing History“, Proceedings of the Cambridge Philological Society 47, 117-140.

v. Möllendorff 2006: Peter von Möllendorff, Lukian. Gegen den ungebildeten Büchernarren. Ausgewählte Werke, übersetzt, Düsseldorf/Zürich.

Porod 2013: Robert Porod, Lukians Schrift „Wie man Geschichte schreiben soll“. Kommentar und Interpretation, Wien 2013.

Schmitz 1997: Thomas A. Schmitz, Bildung und Macht: Zur sozialen und politischen Funktion der zweiten Sophistik in der griechischen Welt der Kaiserzeit (Zetemata 97), München.

Swain 1996: Simon Swain, Hellenism and Empire. Language, Classicism, and Power in the Greek World, Oxford.

Vogt-Spira 1991 = Gregor Vogt-Spira, „Vox und Littera. Der Buchstabe zwischen Mündlichkeit und Schriftlichkeit in der grammatischen Tradition", Poetica 23, 295-327.

Whitmarsh 2001: Tim Whitmarsh, Greek Literature and Roman Empire. The Politics of Imitation, Oxford.

Whitmarsh $2005=$ Tim Whitmarsh, The Second Sophistic, Oxford. 